MÁSTER EN PSICOLOGÍA DE LA SALUD, EVALUACIÓN

Y TRATAMIENTOS PSICOLÓGICOS

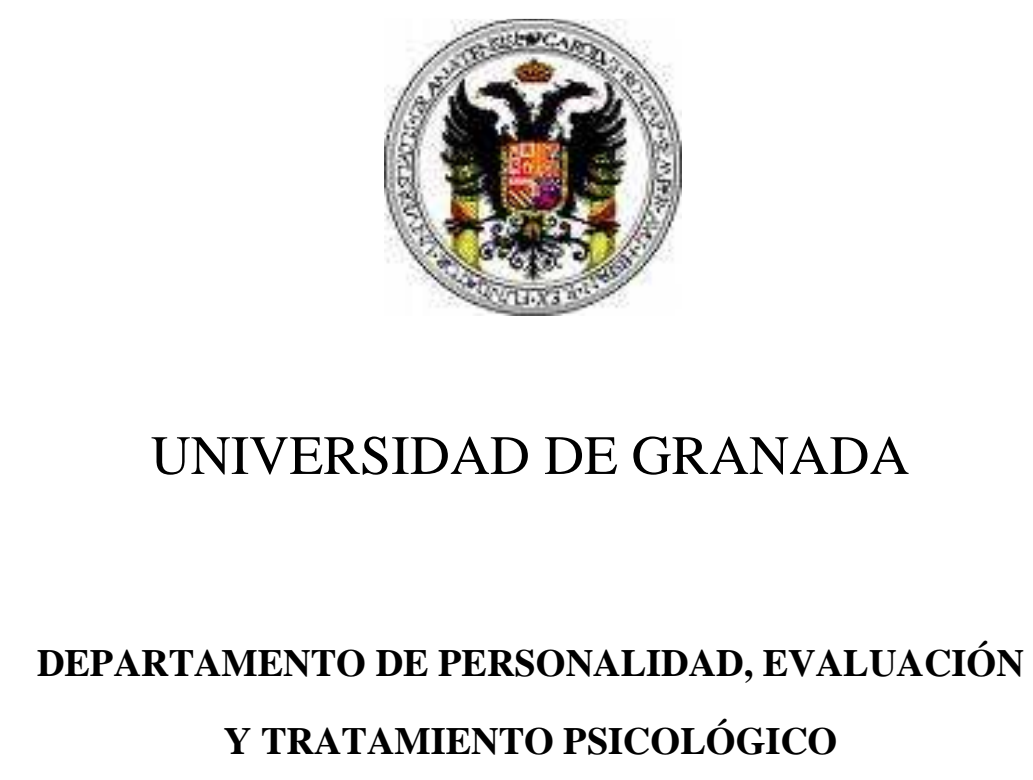

Y TRATAMIENTO PSICOLÓGICO

Trabajo de Investigación:

RELACIONES INTERPERSONALES EN LA ADOLESCENCIA: IMPLEMENTACIÓN DE UN PROGRAMA DE ENTRENAMIENTO EN ASERTIVIDAD Y HABILIDADES SOCIALES PARA ADOLESCENTES DE $1^{\circ}$ Y $2^{\circ}$ DE LA ESO.

AUTOR:

RAFAEL FCO. DELGADO RODRÍGUEZ

DIRECTORAS:

Ma NIEVES PÉREZ MARFIL, y

HUMBELINA ROBLES ORTEGA

Septiembre de 2010 


\title{
Relaciones interpersonales en la adolescencia: implementación de un programa de entrenamiento en asertividad y habilidades sociales para adolescentes de $1^{\circ} \mathrm{y}$ $2^{\circ}$ de la ESO.
}

\author{
Rafael Fco. Delgado Rodríguez
}

\section{RESUMEN}

Introducción: La adolescencia es un periodo en el que las relaciones con los compañeros van ganando importancia, intensidad y estabilidad, de tal forma que el grupo de iguales pasa a constituir el contexto de socialización preferente, y una importante fuente de apoyo. Para que el adolescente consiga unas buenas relaciones sociales, y desarrolle el sentido de ajuste social, es necesario que posea unas adecuadas habilidades sociales. Aunque estas no siempre se acaban desarrollando, lo que supone más probabilidad de padecer diferentes alteraciones psicológicas. Afortunadamente, el entrenamiento en asertividad y habilidades sociales se ha mostrado eficaz en este campo.

Objetivo: Mejorar el nivel de habilidades sociales en adolescentes con déficits en esta área, y su autoconcepto a través de la implementación de un programa de asertividad y habilidades sociales ajustado a sus necesidades.

Material y métodos: Se ha evaluado los déficits en las relaciones sociales y autoconcepto, de una muestra de 78 alumnos de dos Institutos distintos (de las provincias de Granada y Almería). A partir de los déficits detectados, se ha creado un programa de entrenamiento en asertividad y habilidades sociales, y se ha aplicado a alumnos del IES de Granada. Instrumentos: Escala de Habilidades Sociales EHS de Gismero (2000); escala de Autoconcepto Forma5 AF5 de García y Musitu (1999); y el Cuestionario de Evaluación de Dificultades Interpersonales en la Adolescencia CEDIA de Inglés, Méndez e Hidalgo (2000). Se ha empleado un diseño cuasi-experimental pre-post con grupo control.

Resultados: En relación a los distintos instrumentos de evaluación utilizados, podemos observar una relación inversa entre el EHS y el CEDIA (correlación de Pearson $=-.384, p=0.001$ ) y una relación directa entre EHS y la escala Emocional del AF5 (correlación de Pearson= .303, $p=0.007$ ). En cuanto a los beneficios del entrenamiento, se encuentran diferencias significativas entre las puntuaciones pre y post del Grupo tratamiento: aumenta significativamente la puntuación en la escala EHS $(t=-6.25, p=0.001)$; sin embargo aunque en tres dimensiones del AF5 (académica, emocional y física) y CEDIA se observa una mejora, las diferencias no son significativas.

Conclusión: A la vista de los resultados obtenidos, tras la aplicación del programa se producen mejoras significativas en la conducta asertiva y habilidades sociales de los alumnos, si bien no hemos obtenido una mejoría significativa del autoconcepto. Posiblemente es necesario un proceso más largo para poder observar cambios.

Palabras clave: Habilidades sociales, adolescencia, asertividad, autoconcepto, entrenamiento. 


\title{
Interpersonal relationships during adolescence: implementation of an assertiveness and social skills programme for 1 st and $2^{\text {nd }}$ year of Secondary Education adolescents.
}

\begin{abstract}
Introduction: Adolescence is a period of time when relationships gain privilege, importance, intensity, and stability, so that peer relationships is regarded as the main socialization context, and also a great source of support. The acquisition of adequate social skills become of primary necessity if adolescents are to establish solid and appropriate relationships. However, these social skills do not always come to be fully developed and set adolescents in the view of suffering psychological disorders. Fortunately, assertiveness and social skills training has proved to be highly efficient in this field. Aim: To improve social skills in adolescents who show certain deficit in this area, as well as working in their self-concept by implementing an assertiveness and social skills programme in compliance with their necessities.
\end{abstract}

Materials and methodology: The adolescents of this programme are from two different high schools (from the province of Granada and Almeria), from whom we have evaluated social relationships and self-concept deficit. With these detected deficits as basis, we have created an assertiveness and social skills training programme, which has been further applied to those students from the high school in Granada. Instruments and tools: Gismero's Social Skills Scale, EHS (2000); Garcia and Musitu's Self-Concept Scale Form5 AF5 (1999); and Inglés, Méndez and Hidalgo's Interpersonal Difficulties in Adolescence Evaluation Questionare, CEDIA (2000). A quasi-experimental pre-post design with control group has been applied.

Results: Related to the different evaluation tools which have been used, we can observe that there is an inverse relation between EHS and CEDIA (Pearson's correlation $=-.384, p=0.001$ ) and a direct relation between EHS and the emotional dimension of AF5 (Pearson's correlation $=.303, p=0.007$ ). As for the training benefits, we can find noteworthy differences in the results from the pre and post treatment group: EHS scale marking increases $(t=-6.25, p=0.001)$ on the contrary, although we can observe that three of the AF5 five dimensions (academic, emotional, and physic) and CEDIA also improve, these differences are not so noteworthy.

Conclusion: With the results as evidence, we can say that there is a significant improvement in students' assertiveness and social skills, although we have not obtained the same improvement in their self-concept. This may be possibly due to the fact that a larger period of time is required to observe those changes.

Key words: social skills, adolescence, assertiveness, self-concept, training. 


\section{INTRODUCCIÓN}

La adolescencia ha sido definida por algunos autores como un periodo de transición entre la niñez y la edad adulta, en la que la persona ha de enfrentar un gran número de cambios y desafíos evolutivos (Musitu y Cava, 2003). Durante esta etapa, el adolescente se enfrenta a la consolidación de cambios cognitivos como el pensamiento abstracto (Inhelder y Piaget, 1996), el desarrollo de un sistema de valores propios (Kohlberg, 1973), y la definición de su identidad (Erikson, 1963). Al mismo tiempo, los chicos y chicas se van distanciando y desvinculando de sus padres, y las relaciones con los compañeros van ganando importancia, intensidad y estabilidad, de tal forma que el grupo de iguales va a pasar a constituir un contexto de socialización preferente y una importante fuente de apoyo.

Aunque durante la infancia la familia representa el contexto de desarrollo más importante, en la adolescencia tendrá que compartir con el grupo de iguales su capacidad de influencia, hasta situarse en muchos casos en segunda posición (Harris, 1995). De este modo, el adolescente se expone a un amplio abanico de nuevas situaciones sociales (fiestas, bares, oficinas públicas, establecimientos comerciales, etc.), en las que aumenta las relaciones con personas desconocidas o no allegadas (Flores y Díaz, 1995).

Debido a estos cambios producidos en la adolescencia y lo que con ello conlleva, los comportamientos sociales cobran gran relevancia. El importante rol de estas conductas se evidencia en el papel que juegan en la identificación de psicopatología, especialmente durante esta etapa de la vida. Por ejemplo, la mayoría de las categorías diagnósticas incluidas en el actual sistema de clasificación DSM-IV-TR (American Psychiatric Association, 2000) que son aplicables a niños y adolescentes, incluyen el funcionamiento social como criterio de patología. Aproximadamente el $45 \%$ de los síndromes clínicos del Eje I, y casi todos los problemas de personalidad del Eje II, presentan problemas en el funcionamiento social como un posible 
criterio, y la mayoría de los demás trastornos tienen implicaciones a nivel social (Hansen, Giacoletti y Nangle, 1995).

Las nuevas situaciones y relaciones a las que se enfrenta el adolescente hacen de las interacciones sociales competentes, y de las habilidades sociales necesarias, elementos clave en este periodo de la vida para alcanzar el ajuste y funcionamiento exitoso en sociedad. Numerosos son los estudios que muestran la relación entre ambos componentes y la importancia de los dos en el desarrollo del adolescente. Ya en 1969, O’Connor señalaba que un niño que presentara un importante déficit en habilidades sociales (referidas como HHSS) tendría un serio hándicap en la adquisición del repertorio de conductas necesarias para un funcionamiento social efectivo. El déficit en HHSS está presente muy a menudo entre adolescentes que exhiben problemas de comportamiento disruptivo y externalizantes, tales como la delincuencia (Hansen, Lawrence y Christoff, 1988), y además se asocia con problemas internalizantes como depresión o ansiedad (Christoff, Scott, Kelley, Schlundt, Baer y Kelly, 1985; Hansen, Nangle y Meyer, 1998). Problemas adicionales asociados con el déficit en HHSS incluyen abuso de sustancias, ofensas sexuales, soledad, embarazos no deseados y enfermedades de transmisión sexual (Hansen et al., 1995). Tales habilidades son necesarias para el establecimiento de unas buenas relaciones sociales y de amistad durante la adolescencia, y a su vez, estas relaciones han mostrado ser importantes para la autoestima y el sentimiento de satisfacción vital del adolescente (Chou, 2000).

De todo lo mencionado anteriormente, uno de los problemas de ajuste más importantes que se producen en la adolescencia como causa de un déficit de HHSS, y que los autores han mencionado a lo largo de los años, es la experiencia de soledad. Esta se produce como consecuencia de no satisfacer la necesidad de intimidad y auto-revelación que se ven incrementadas en la adolescencia, para lo cual es necesario las relaciones cercanas con el grupo de iguales (Brennan, 1982). El sentimiento de soledad crónica puede suponer un riesgo para 
los adolescentes en lo que se refiere al desarrollo de problemas emocionales severos, por ejemplo, la soledad en la adolescencia se ha relacionado con tasas de depresión (Kaiser y Berndt, 1985; Moore y Schultz, 1983), suicidio (Tabachnick, 1981) y anorexia nerviosa (Gilbert y DeBlassie, 1984).

El bajo rendimiento académico también ha sido investigado como consecuencia de las dificultades interpersonales, ya que éstas promueven en los adolescentes una escasa participación en clase, resistencia a presentar trabajos en público y la tendencia a evitar preguntar al profesor, lo que impediría la aclaración de dudas (Francis y Radka, 1995); además, la evitación de las relaciones con los compañeros genera sentimientos de aislamiento (Walter e Inderbitzen, 1998), y la correspondiente baja tasa de reforzamiento social origina depresión (Francis, Last y Strauss, 1992). Además de lo expuesto anteriormente, los déficits de HHSS también conducen a una relación disfuncional con los padres, que impide la comunicación, negociación y resolución de conflictos efectiva (Openshaw, Mills, Adams y Durso, 1992).

Por lo tanto, las consecuencias negativas derivadas de un déficit de HHSS en la adolescencia son evidentes, y parece claro que aquellos adolescentes que muestran una mayor competencia para establecer relaciones con los compañeros presentan un mejor ajuste emocional y conductual (Sánchez-Queija y Olivo, 2003). Esta relación entre el déficit en HHSS y los problemas de ajuste es bidireccional, ya que un déficit en HHSS puede llevar al adolescente a sufrir problemas de ajuste, y en consecuencia, estos problemas de ajuste limitarán al adolescente en el desarrollo de HHSS (Hansen et al., 1995).

Son diversos los enfoques que tratan de explicar cómo los niños y niñas desarrollan las habilidades interpersonales necesarias para conseguir adherirse a un grupo de amigos y, así, beneficiarse de todo lo que esto supone. No hay datos definitivos sobre cómo y cuándo se aprenden las habilidades sociales, pero la niñez es sin duda un período crítico (Caballo, 2009). Desde las teorías más biologicistas, se ha hablado de que los niños pueden nacer con un sesgo 
temperamental, y que su manifestación conductual se relacionaría con un sesgo fisiológico heredado que podría mediar la forma de responder al comportamiento de los demás. De acuerdo con esta idea, el temperamento de los niños y niñas al nacer es el que va a determinar la naturaleza del ambiente socioemocional interpersonal en muchos aspectos y, de esta manera, determina también la facilidad para el aprendizaje; con las demás condiciones iguales, el individuo emocionalmente expresivo tiende a crear para él un ambiente social y emocionalmente más rico (Buck, 1991). El temperamento va a condicionar la expresividad del menor, y esta expresividad facilita el desarrollo de las habilidades sociales y fomenta la competencia social (Buck, 1991). Es decir, los niños más inhibidos van a disponer de menos oportunidades de aprender y practicar las conductas sociales, y probablemente reciban menos refuerzos (alabanzas, sonrisas, caricias, etc.) por las personas de su entorno, ocurriendo lo contrario con los niños más desinhibidos (Caballo, 2009).

Sin embargo, incluso en situaciones donde el comportamiento está determinado en gran medida por lo biológico, especialmente en las primeras experiencias sociales, en la mayoría de las personas, el desarrollo de las habilidades sociales dependerá principalmente de la maduración y de las experiencias de aprendizaje (Argyle, 1969).

Para Bellack y Morrison (1982) la teoría del aprendizaje social es la que explica de forma más aceptada cómo los niños y niñas se convierten en seres sociales. Ya desde la primera infancia, niños y niñas observan la interacción de sus padres con ellos, así como con otras personas, y aprenden su estilo (tanto la conducta verbal como la no verbal). Desde la teoría del aprendizaje social se contempla que, en la familia, se aprenden patrones conductuales, estilos relacionales y habilidades sociales a través del modelado e imitación del comportamiento de los progenitores, que posteriormente generalizarán a otros contextos de desarrollo, como el grupo de iguales (Bandura, 1982; Sánchez-Queija y Oliva, 2003). Aunque es cierto que el mal funcionamiento social en la edad adulta (o una habilidad social apropiada) 
no depende totalmente de los padres, sino que el grupo de iguales es un importante modelo y fuente de reforzamiento, especialmente durante la adolescencia (Caballo, 2009), se ha de seguir aprendiendo para continuar siendo socialmente habilidoso. Esta última conclusión es apoyada por datos de autores como Morgenson, Reidor y Campion (2005), los cuales aseguran que la falta de motivación y reforzamiento en el uso de las habilidades sociales, poco a poco, lleva a los individuos a la pérdida de conductas habilidosas y una disminución en la socialización.

Por otro lado, autores tan destacados como Bowlby (1979), desde la teoría del apego señalan la importancia que tienen los vínculos de apego creados con los padres durante la infancia, para el establecimiento de posteriores relaciones afectivas, de forma que, aquellos niños y niñas que establecieron relaciones de apego seguro con unos padres que se mostraron cariñosos y sensibles a sus peticiones, serán más capaces de establecer relaciones con los iguales caracterizados por la intimidad y el afecto.

Sin restar importancia a ninguno de estos enfoques, lo que parece claro es que, el hecho de que los niños y niñas aprendan las "normas sociales" básicas que les permitirán tener un buen desempeño social en la adolescencia y en la edad adulta, atiende a un ambiente que se escapa a su control. Es decir, se ha de dar una situación a nivel biológico que capacite al menor para ser expresivo, y así interactuar con el ambiente de forma que éste le sea reforzante, ha de tener la fortuna de nacer en un seno familiar donde sus progenitores hagan gala de unas buenas habilidades sociales y, además, han de relacionarse en un ambiente social que les enseñe la forma de interactuar con otras personas de forma adecuada. Cuando estos factores coexisten, podemos tener a un niño o niña que presenta habilidades sociales para relacionarse de forma adecuada en las diversas situaciones que van a aparecer en su vida. No obstante, la pregunta que surge es qué ocurre cuando falta/n alguna/s de estas condiciones.

Por desgracia, como hemos visto, el aprendizaje de las habilidades interpersonales no se encuentra institucionalizado formalmente, es decir, no se estudia en la escuela como cualquier 
otra asignatura, como por ejemplo las matemáticas, en cuyo caso hay una persona especializada que ha pasado años de su vida estudiando esta materia en la universidad para después transmitirla a los alumnos. En el caso de las HHSS no hay una asignatura específica que nos enseñe qué tipo de comportamientos son adecuados en una situación, y cuáles en otra. Por el contrario, se les permite a los menores que sean ellos quienes se dirijan a sí mismos en la ardua tarea de convertirse en seres sociales, incluso cuando en algunas ocasiones, el ambiente en el que se desenvuelven es totalmente anómalo para este aprendizaje. Pero por fortuna, la conducta interpersonal se aprende y, por tanto, puede enseñarse y modificarse. Sería por ello deseable que, desde los centros educativos, se prestara la posibilidad a aquellos niños y adolescentes que presenten estos déficits en habilidades sociales, de acceder a un programa que les permitiese mejorar la capacidad de relacionarse con las personas de su entorno. Aún habiéndose desarrollado programas de este tipo en algunos centros, y habiendo mostrado sus beneficios, no deja de ser algo fortuito, no establecido de forma general en todos los centros.

\section{Objetivo de la investigación}

Vista la importancia que supone tener unas buenas HHSS en la adolescencia, donde la identificación y el sentimiento de pertenencia al grupo de iguales se tornan en aspectos de los más importantes para la supervivencia emocional y psicológica del adolescente $\mathrm{y}$, dado que diferentes programas han mostrado su efectividad en esta área de la Psicología, el presente trabajo abarca dos propósitos, que son comentados a continuación.

Uno de los objetivos de nuestra investigación ha sido centrarnos en el análisis de algunas variables psicosociales, íntimamente relacionadas con el bienestar humano, como son el autoconcepto, la asertividad y las habilidades para la interacción social en alumnos de la ESO. Hemos querido comprobar si dichas variables están relacionadas (autoconcepto, asertividad y habilidades sociales). 
El segundo de los objetivos de nuestra investigación, fue el de elaborar y aplicar un programa de entrenamiento en asertividad y HHSS, a partir de los déficit observados. Se trataría de comprobar si los alumnos que realicen este programa, mejoran en asertividad y HHSS, y si el programa incide también en los niveles de autoconcepto.

Con respecto a este segundo propósito, son diversos los autores que muestran que este tipo de programas logran alcanzar todos sus objetivos y tener efectos beneficiosos, siempre y cuando el programa se aplique de manera continua y cumpliendo con el protocolo científico con el que fue elaborado (Frederick y Morgenson, 2005; Nas, Brugman y Koops 2005; Llanos Baldivieso, 2006). Otros como Monjas (2002) sostienen que, después de la aplicación de un programa de enseñanza de HHSS, los participantes tendrán mejores herramientas para poder actuar de manera asertiva con su grupo de pares y con las personas adultas que lo rodean. En un meta-análisis realizado por Rosa, Ingles, Olivares, Espada, Sánchez-Meca y Méndez (2002) se demuestra que el entrenamiento en HHSS tiene una eficacia global moderada $(d+=0.62)$, coincidiendo estos datos con meta-análisis previos en este campo (Beelmann, Pfingsten y Losel, 1994; Denham y Almeida, 1987; Schneider, 1992), y dicha eficacia aumenta durante el seguimiento $(\mathrm{d}+=1.25)$. Por lo tanto, los autores concluyen que, el entrenamiento en general tiene éxito, produciéndose los mejores resultados en las variables de competencia social (asertividad, afrontamiento y habilidades sociales), frente a las variables más periféricas (autoestima y ansiedad). Por último, en Caballo (2009) podemos leer "el entrenamiento en habilidades sociales se encuentra entre las técnicas más potentes y más frecuentemente utilizadas para el tratamiento de los problemas psicológicos" (p.181).

A modo de resumen, podemos señalar que el objetivo de nuestra investigación es, por un lado, analizar las necesidades de alumnos de dos IES públicos en relación a las HHSS y autoestima, y por otro lado, aplicar un programa de entrenamiento en asertividad y habilidades sociales para alumnos de los primeros cursos de la ESO con déficit en HHSS. 
Las hipótesis principales que pretendemos poner a prueba en el presente estudio son las siguientes:

HIPÓTESIS I: Esperamos encontrar correlación entre asertividad/habilidades sociales y dificultades interpersonales. De forma que, aquellos alumnos que presenten déficit en asertividad/HHSS tendrán más dificultades en las relaciones interpersonales.

HIPÓTESIS II: Esperamos encontrar relación entre las variables de asertividad y HHSS y autoconcepto, de forma que, los alumnos que presenten déficit en HHSS/asertividad también muestren un autoconcepto más bajo.

HIPÓTESIS III: Esperamos encontrar mejoras significativas en HHSS y disminución de las dificultades interpersonales al comparar las evaluaciones pre y post de los adolescentes que participen en la condición experimental. Por otro lado, no se espera que se den ningún cambio en las puntuaciones pre y post de aquellos participantes que pertenezcan a la condición control.

HIPÓTESIS IV: Aunque el entrenamiento se ha centrado en la mejora de la asertividad y HHSS de los alumnos, se espera encontrar una mejora en el autoconcepto. Se hipotetiza que el aumento de estas habilidades en los adolescentes mejora su competencia a nivel social, lo que podría mejorar su autoconcepto y beneficiar su autoestima. En lo referente al grupo control, no se espera ningún cambio en el autoconcepto.

\section{MATERIAL Y MÉTODO:}

\section{Participantes:}

La muestra sobre la que se realizó el estudio, estuvo formada por un total de 78 adolescentes (35 chicos y 43 chicas) de edades comprendidas entre los 12 y los 16 años $($ Media $=12.92$ y Desviación Típica $=0.94)$, que asistían a centros educativos públicos de la provincia de Granada y Almería (43 alumnos del IES de la provincia de Granada, el 54.4\%, y 
36 al de Almería, el 45.6\%). Fueron seleccionados estos dos centros educativos gracias a la disponibilidad que teníamos de acceder a los alumnos. Los adolescentes pertenecían a $1^{\circ}$ y $2^{\circ}$ de la ESO (el $66.7 \%$ de los alumnos cursaban $1^{\circ}$ de ESO y el $33.3 \%$ cursaban $2^{\circ}$ de ESO).

Del conjunto de los alumnos de la provincia de Granada, se seleccionó una muestra de chicos y chicas para llevar a cabo el segundo propósito de nuestra investigación, la realización y aplicación de un programa de asertividad y habilidades sociales y la posterior evaluación de su eficacia. El criterio de selección fue que los alumnos tuvieran puntuaciones en la Escala de Habilidades Sociales (EHS) por debajo del Centil 40.

\section{Instrumentos}

- Escala de Habilidades Sociales (EHS) (Gismero, 2000).

El EHS es una escala que evalúa conducta asertiva y habilidades sociales. Está compuesto por 33 ítems, 28 de los cuales están redactados en el sentido de falta de aserción o déficit en habilidades sociales y 5 de ellos en el sentido positivo. Se contesta en formato Likert de 4 puntos, desde "No me identifico en absoluto; la mayoría de las veces no me ocurre o no lo haría" (A) a "Muy de acuerdo y me sentiría o actuaría así en la mayoría de los casos" (D). La puntuación total oscila entre 33 y 134, donde no hay puntos de corte, sino que a mayor puntuación global, más habilidades sociales y capacidad de aserción en distintos contextos.

Este instrumento evalúa las diferentes dimensiones del constructo de la conducta asertiva / habilidades sociales (Caballo, 1989; Caballo y Buela, 1988; Carrasco, Clemente y Llavona, 1983; Galassi y Galassi, 1980, Gismero, 2000). En concreto, se han aislado 6 factores: 1) autoexpresión en situaciones sociales, 2) defensa de los propios derechos como consumidor, 3) expresión de enfado o disconformidad, 4) decir no y cortar interacciones, 5) hacer peticiones, y 6) iniciar interacciones positivas con el sexo opuesto. 
Se ha constatado que el EHS presenta una alta consistencia interna, tal y como se expresa en un coeficiente de fiabilidad alto ( $a l f a=0.88)$, y adecuada validez convergente (Gismero, 2000).

- Autoconcepto Forma 5 (AF5) (García y Musitu, 1999).

El AF5 evalúa el autoconcepto de la persona en sus 5 dimensiones (académico/laboral, social, emocional, familiar y físico), considerando 6 reactivos por dimensión. La escala que presenta este instrumento se distribuye del 1 al 99, donde los participantes tienen un gran rango para responder según lo de acuerdo que estén con cada ítem. No existe una puntuación total en la escala que nos indique el autoconcepto de la persona, por el contrario, éste se calcula por las diferentes dimensiones que lo forman, haciendo referencia a la teoría de la dimensionalidad del autoconcepto (modelo teórico de Shavelson, Hubner y Stanton, 1976), en la que se basa la construcción de esta escala. El AF5 presenta una estructura dimensional, formada por las 5 dimensiones más representativas del autoconcepto. No existe punto de corte para las puntuaciones de cada dimensión, sino que a mayor puntuación mejor autoconcepto.

Se ha constatado que los ítems presentan una adecuada fiabilidad en términos de consistencia interna, tanto por factores como en la escala total, lo que facilita su aplicación en diversos contextos (García y Musitu, 1999; Tomás y Oliver, 2004). La consistencia interna de los 30 reactivos fue de 0.84 , de la dimensión académica/laboral, 0.89 , de la social, 0.73 , de la emocional, 0.73, de la familiar, 0.80, y del físico, 0.78 .

La validez de esta estructura multidimensional definida a priori se ha constatado empíricamente con análisis factoriales exploratorios en muestras de España (García y Musitu, 1999), lo que permite concluir, que la escala tiene, a las luces de la evidencia empírica analizada, una buena calidad psicométrica (Tomás y Oliver, 2004). 
- Cuestionario de Evaluación de Dificultades Interpersonales en la Adolescencia (CEDIA) (Ingles Saura, Méndez e Hidalgo, 2000).

Se trata de una medida de autoinforme destinada a detectar el nivel de dificultad que presentan los adolescentes de entre 12 y 18 años en sus relaciones interpersonales. Está compuesta por 39 ítems distribuidos en 5 factores: 1) Aserción, 2) Relaciones con el Otro Sexo, 3) Relaciones con Iguales, 4) Hablar en Público, y 5) Relaciones Familiares. Estos factores son los considerados por muchos investigadores como los más importantes durante la adolescencia (Bracken y Crain, 1994; Cavell y Felley, 1994). Las preguntas muestrean una amplia gama de relaciones interpersonales con personas de diferente edad, género, nivel de autoridad y grado de conocimiento o confianza, en diversos contextos, hogar, colegio, compañeros del mismo o de distinto sexo y situaciones de calle, comerciales o de servicios. El adolescente valora su grado de dificultad de acuerdo con una escala Likert de 5 puntos $(0=$ ninguna dificultad; 4= máxima dificultad). La puntuación total oscila entre 0 y 156, de modo que una mayor puntuación es signo de una mayor dificultad interpersonal.

Ingles Saura, Méndez e Hidalgo (2000) demostraron que el CEDIA presenta una consistencia interna en la puntuación total de una $\alpha$ de Cronbach $=0.91$, y una fiabilidad testretest de 0.78 (en un intervalo de dos semanas). La consistencia interna de los factores de la prueba es de 0.86 para Aserción, 0.82 para las Relaciones con el Otro Sexo, de 0.75 para Relaciones con Iguales, de 0.78 para Hablar en Público, y de 0.69 para las Relaciones Familiares. Por lo tanto, esta escala posee adecuadas propiedades psicométricas, elevada fiabilidad y estructura dimensional (validez de constructo), así como viabilidad, ya que el tiempo de aplicación se reduce a 15-20 minutos. 


\section{Procedimiento:}

La actuación en cada uno de los centros fue distinta. En el IES de la provincia de Granada se contactó con la Orientadora, a la que se le comentó la investigación, así como el propósito de la misma. Ésta lo puso en conocimiento del equipo directivo, con el que concertamos una cita.

Cuando se obtuvo el permiso del equipo directivo se les entregó una carta para los padres del grupo de alumnos a evaluar, de $1^{\circ}$ y $2^{\circ}$ de la ESO, donde se explicaba el objetivo de la evaluación, y se les adjuntaba una autorización.

Una vez dispusimos de las autorizaciones firmadas, se entrenó al Jefe de Estudios para que aplicara los cuestionarios, y así no interrumpir con la dinámica del instituto. Recogidos los datos, se hizo una selección de los alumnos que presentaban puntuaciones significativas en la Escala de Habilidades Sociales (EHS), que fueron convocados para explicarles en qué iba a consistir el entrenamiento, y así darles la oportunidad de acudir voluntariamente. Acudieron 12 alumnos de 29 que había convocados (3 chicos y 9 chicas), y todos, excepto un chico, decidieron participar en el programa. Finalmente, a la primera sesión no acudieron dos chicos, y otras 2 chicas se fueron descolgando en la segunda y tercera sesión, de tal manera que el grupo quedó constituido por 7 chicas. Al terminar el entrenamiento se volvió a evaluar a este grupo experimental y se convocó a 7 alumnos que habían tenido puntuaciones deficitarias en el EHS (Centil inferior a 40), y no habían recibido entrenamiento, para formar el grupo control.

En lo que respecta al IES de la provincia de Almería, se contactó con la profesora de Inglés de $1^{\circ} \mathrm{ESO}$, y ésta se encargó de poner en conocimiento de la directiva nuestro propósito. Una vez se obtuvo el permiso, y disponíamos de las autorizaciones firmadas, se procedió a entrenar a la profesora para que posteriormente administrara los cuestionarios. Recogidos los datos, hemos realizado la selección de los alumnos que presentan puntuaciones significativas en la Escala de Habilidades Sociales, y en breve serán convocados para explicarles en qué va a 
consistir el entrenamiento, y así darles la oportunidad de acudir voluntariamente al programa, dado que por diversas razones no se ha podido llevar a cabo al mismo tiempo que el grupo de Granada.

El estudio se desarrollo en tres fases:

Fase uno: Evaluación global inicial, llevada a cabo tres semanas antes del comienzo del programa de entrenamiento. Una vez obtenidos los datos de los alumnos y habiendo convocado a los que presentaban déficit en el EHS, se les informó del objetivo del programa y se les dió la oportunidad de comenzar el entrenamiento.

Fase dos: Intervención, aplicación del programa de entrenamiento en asertividad y habilidades sociales diseñado a partir del análisis de necesidades del alumnado. El programa ha sido estructurado en 6 sesiones (a razón de una sesión por semana), de 1 hora de duración cada una. Esta fase no está cerrada, ya que hemos llevado a cabo la intervención en el centro educativo de Granada, y nos falta realizarlo en el centro educativo de Almería.

Fase tres: Esta fase solo se llevó a cabo con los alumnos que pertenecían a los grupos de tratamiento y control, ya que se trataba de la evaluación postratamiento, realizada una semana después de la última sesión del programa.

A continuación exponemos el programa aplicado y sus características más importantes.

- Programa de entrenamiento en asertividad y habilidades sociales DEAA:

Gracias a la posibilidad de poder analizar a priori las características de la población diana, pudimos construir un programa integrado por las habilidades más deficitarias de este grupo. Así eliminamos las barreras con las que muchos programas de HHSS se encuentran, y pudimos ajustar nuestro trabajo lo máximo posible a los alumnos.

Antes de comenzar a diseñar el programa, nuestro propósito era que se tratara de una actividad divertida, en la que los alumnos que asistieran voluntariamente se lo pasaran bien y, 
consiguieran pasar una hora de entretenimiento, tanto con el profesional que llevara a cabo el programa, como con los compañeros. Esta es una característica muy importante teniendo en cuenta que el programa se desarrollaba por la tarde, en hora extra-escolar y era totalmente voluntario. Nos propusimos que, además de divertido fuera educativo, ya que el objetivo del programa es que lo alumnos aprendan a desenvolverse de forma habilidosa en situaciones sociales.

En cuanto a la forma de desarrollarlo, las clases magistrales se evitaron en todo lo posible, de hecho, los alumnos estaban sentados en círculo y la dinámica de clase se intentó que fuera lo más aplicada posible, a través de realización de juegos, ejercicios en grupo, representación de papeles, exposiciones, etc.

Por último, el programa debía ser adaptado al tiempo en el que vivimos, es decir, consideramos que una forma de "enganchar" a los adolescentes era tratar las habilidades sociales y la asertividad utilizando temas de actualidad para ellos, con personajes, series y noticias que sean importantes y significativos. Todo estos componentes del programa eran importantes para inducir una actitud positiva y entusiasta en los menores, ya que para este tipo de entrenamiento se requiere de la participación activa por parte de los usuarios del programa, para lo cual es necesaria su motivación.

Estas cuatro características que hemos señalado anteriormente (Divertido, Educativo, Aplicado y Actualizado), son las que dan nombre a nuestro programa (programa de entrenamiento en asertividad y habilidades sociales DEAA).

Para dar forma al programa, en cuanto a contenido y actividades a realizar, revisamos varios programas de entrenamiento (Camacho Gómez y Camacho Calvo, 2005; Fernandez Berrocal y Melero Zabal, 1995; Garaigordobil Landazabal, 1996; Garaigordobil Landazabal, 2001; García Pérez y Magaz Lago; Gil y Leon, 1998; Monjas, 2009; Riesco González, 2005; Roca, 2007; Vaello Orts, 2005; 1998; Vallés Arándiga y Vallés Tortosa, 1994; Vera Guerro y 
Roldán Maldonado, 2009). De algunos de ellos cogimos las actividades que nos parecieron más dinámicas y se ajustaban al déficit del grupo. Muchas de estas fueron modificadas y adaptadas para hacerlas lo más dinámicas posibles, y otras fueron desarrolladas por el autor del trabajo.

Para conseguir estas condiciones, hemos utilizado técnicas cuya efectividad está demostrada para el desarrollo de las sesiones, estas son: instrucción, modelado, representación conductual, retroalimentación del rendimiento, reforzamiento y generalización o fase de tareas (Wilkinson y Canter, 1982; Caballo, 2009).Sin embargo, también se introdujo el elemento audiovisual con la ayuda del programa Power Point@ como soporte para desarrollar las sesiones, lo que nos permitía ejemplificar en todo momento lo que estábamos hablando, además de conseguir captar la atención de los menores por el dinamismo de las diapositivas. Este aspecto es importante, tal y como muestra Rosa (Rosa et al., 2002) en su meta-análisis, el hecho de que la información llegue de forma oral y diferida (mixta), repercute más positivamente en los resultados que si llega solo de forma oral, ya que cuanto más fuentes se utilicen para mostrar una conducta, más fácilmente será adquirida.

Para Lange (1981, Lange, Rimm y Loxley, 1978), el entrenamiento en HHSS ha de pasar por cuatro etapas, que fueron las consideradas en nuestro entrenamiento, éstas son: 1.Desarrollar en el individuo unas creencias de respeto hacia sus propios derechos personales y de los demás, 2.- distinción entre conductas asertivas, no asertivas y agresivas, 3.reestructuración cognitiva y, 4.- ensayo conductual de respuestas asertivas en situaciones determinadas. Quizás la diferencia de la estructura que planteaban estos autores con la desarrollada en nuestro programa, esté en que nosotros no hemos hecho énfasis en la reestructuración cognitiva, sino que nos hemos centrado en la relación entre pensamientoemoción, y en la importancia de considerar una situación desde diferentes puntos de vista. Además, para Lange (1981) la instrucción, el modelado, el ensayo conductual, el feedback, el 
reforzamiento y la generalización son técnicas de la cuarta etapa, mientras que nosotros las hemos utilizado en todas ellas.

Objetivo del programa: El objetivo general es aumentar la competencia social de los adolescentes con su grupo de iguales y adultos, para así mejorar su sentido de pertenencia al grupo y ajuste social, repercutiendo positivamente en la mejora de su autoconcepto, y por lo tanto en su autoestima, al disfrutar de sus interacciones sociales y sentirse valorados y respetados dentro del grupo. Este objetivo se consigue a través de una serie de sub-objetivos: a) hacer conscientes a los adolescentes de la importancia del grupo de iguales para su bienestar emocional, y en consecuencia, de la importancia de las HHSS para un buen desempeño social; b) darles las herramientas para que sean capaces de identificar qué tipo de estilo de conducta social están teniendo en situaciones sociales, promoviendo un comportamiento asertivo, y que además, identifiquen el estilo de las personas que interactúan con ellos y sepan cómo responder asertivamente ante personas o situaciones que les hacen sentir mal y; c) a nivel más cognitivo, se intenta que los adolescentes sean capaces de analizar las situaciones y problemas sociales desde distintos puntos de vista, haciendo énfasis en que piensen antes de actuar.

Destinatarios del programa: Está diseñado para el entrenamiento con adolescentes de entre 12 y 16 años, tanto para aquellos sin problemas aparentes como para los que presentan problemas internalizantes (aislamiento y retraimiento social, timidez, baja autoestima, etc.) o problemas externalizantes (agresividad, hostilidad, etc.), sin que estén presentes otros problemas más graves como fobia social, depresión grave, etc., para cuyo caso se requeriría este programa como complementario al tratamiento de elección.

En el Anexo I, se recoge las sesiones y los contenidos que hemos desarrollado y trabajado con las adolescentes a lo largo del programa. 


\section{RESULTADOS}

Este apartado lo hemos clasificado siguiendo los diferentes objetivos de la investigación. Por un lado, hemos procedido al análisis descriptivo de las variables sociodemográficas y las psicológicas de la muestra total de alumnos, y por otro, se ha realizado el mismo análisis de las variables socio-demográficas y psicológicas en el grupo de alumnos en el que se ha desarrollado el entrenamiento, y en el grupo control. Además, como parte del segundo objetivo, se ha evaluado el efecto del programa aplicado.

Los análisis estadísticos aplicados a las variables socio-demográficas y a las psicológicas, han sido, análisis descriptivos, pruebas $t$ para muestras relacionadas $\mathrm{e}$ independientes, pruebas no paramétricas (chi cuadrado), y correlaciones. En la comprobación del efecto del programa, se ha seguido un diseño cuasi-experimental (de caso típico) pre/post con grupo control. Todos los análisis estadísticos han sido realizados con el programa SPSS 15.0 (Stadistical Packaged for Social Sciences).

\section{Muestra Total}

Variables Socio-Demográficas:

\section{- Análisis Descriptivos}

En primer lugar, se procedió a realizar un análisis descriptivo de las variables sociodemográficas de la muestra total, formada por los alumnos de ambos IES. Como se ha señalado anteriormente, la edad de los participantes se distribuía entre los 12 y los 16 años (Media= 12.92; D.T.=0.94). El 37.2\% de los menores tenía 12 años en el momento de la evaluación, $41 \%$ tenía 13 años, $16.7 \%$ de los adolescentes 14 años, $2.6 \%$ tenía 15 años, y el mismo porcentaje, $2.6 \%, 16$ años.

Con respecto a la variable repetidor, donde señalaban si habían repetido o no, y en el caso de haberlo hecho, cuántas veces, 60 alumnos no habían repetido (76.9\%), 13 adolescentes lo 
habían hecho una vez (16.6\%), y solo 5 menores habían repetido dos veces, el $6.4 \%$ de la muestra total.

Otra de las variables socio-demográficas incluidas en nuestra investigación, fue el nivel de estudios de los padres de los alumnos que participaron en la evaluación. Los menores cuyos padres no tenían ningún estudio era del $2.6 \%$, el $43.6 \%$ de los menores, tenían padres que habían cursado estudios primarios, 38.5\% tenían padres con estudios secundarios, y solo el 15.4\% tenía padres con estudios universitarios. En cuanto a las madres de los adolescentes, el $2.5 \%$ no tenía estudios, $22.5 \%$ estudios primarios, el $60 \%$ habían cursado estudios secundarios, y el $15 \%$, poseía estudios universitarios.

La última variable socio-demográfica evaluada, fue el estado civil de los padres de los menores. La distribución de porcentajes fueron, el $88.5 \%$ de los padres estaban casados, el $9 \%$ separados/divorciados, y solo el $2.6 \%$ viudos/as.

\section{Variables Psicológicas:}

\section{- Análisis Descriptivos}

Las variables psicológicas evaluadas fueron, la conducta asertiva y las habilidades sociales (EHS), las dificultades en las relaciones interpersonales (CEDIA), y el autoconcepto (AF5). La Media y D.T. se recogen en la tabla 1.

Como ya se ha comentado, para formar parte de los grupos de tratamiento y control, seleccionamos a los alumnos con un Centil 40 o inferior en la escala EHS. De la distribución de la muestra total cabe destacar que casi la mitad de la muestra, el $42.31 \%$ de los alumnos, presentaron una puntuación total que está por debajo del Centil 30. Este porcentaje aumenta hasta el $64 \%$ si tenemos en cuenta a todos los alumnos cuya puntuación se encuentra por debajo del Centil 50.

Como la muestra total estaba compuesta por alumnos de dos IES distintos, hemos realizado también los análisis descriptivos de ambos institutos por separado (ver tabla 1). Se 
realizó una prueba $t$ para muestras independientes, y así, comprobar si existían diferencias significativas entre ambas poblaciones de alumnos.

Los resultados muestran que no existen diferencias significativas en la variable conducta asertiva y habilidades sociales (EHS), en la variable de dificultad en las relaciones interpersonales (CEDIA), ni en cuatro de las cinco dimensiones que miden el autoconcepto. Encontrándose la única diferencia significativa en la dimensión familiar del AF5, donde los participantes de Almería, presentan una mejor puntuación que los de Granada, lo que muestra un autoconcepto familiar más alto (ver tabla 1).

Tabla 1. Media y Desviaciones Típicas de la escala EHS, CEDIA y las 5 dimensiones del AF5, para los alumnos de los IES de Granada y Almería.

\begin{tabular}{|l|c|c|c|c|c|}
\hline & \multicolumn{3}{|c|}{ IES } & \\
\cline { 2 - 6 } & $\begin{array}{c}\text { Muestra Total } \\
\text { Media (D.T.) }\end{array}$ & $\begin{array}{c}\text { Granada } \\
\text { Media (D.T.) }\end{array}$ & $\begin{array}{c}\text { Almería } \\
\text { Media (D.T.) }\end{array}$ & $\boldsymbol{t}$ & $\boldsymbol{p}$ \\
\hline CEDIA & $89.89(11.35)$ & $89.79(11.53)$ & $90.03(11.30)$ & -.091 & .927 \\
\hline AF5 Acade/labo & $50.73(22.16)$ & $51.79(23.43)$ & $49.43(20.77)$ & .466 & .643 \\
\hline AF5 Social & $7.08(1.83)$ & $6.88(1.89)$ & $7.33(1.76)$ & -1.069 & .288 \\
\hline AF5 Emocional & $5.66(1.41)$ & $7.54(1.31)$ & $7.81(1.53)$ & -.845 & .401 \\
\hline AF5 Familiar & $8.67(1.48)$ & $8.36(1.63)$ & $9.05(1.18)$ & -2.102 &. $\mathbf{0 3 9} *$ \\
\hline AF5 Físico & $6.00(1.79)$ & $5.92(1.76)$ & $6.09(1.77)$ & -.422 & .675 \\
\hline
\end{tabular}




\section{- Correlaciones}

Así mismo, se realizó un análisis de correlaciones con la finalidad de determinar la relación entre las diversas variables evaluadas en los participantes. Para la muestra total, se observa que la escala EHS correlaciona de forma significativa con el cuestionario CEDIA (correlación de Pearson= -.384; sig= .001). La relación entre ambas es negativa, lo que significa, que a mayor habilidades sociales posea el menor, menos dificultades tendrá en sus relaciones interpersonales. El EHS, también correlaciona de forma significativa con la dimensión emocional del AF5 (correlación de Pearson= .303; sig= .007), por lo que, cuanto más hábil socialmente sea el adolescente, mejor autoconcepto emocional. Además, se ha encontrado correlación significativa, y negativa, entre el CEDIA y dos dimensiones del AF5; la dimensión emocional (correlación de Pearson= -.327; sig= .003), y la física (correlación de Pearson $=-.238 ; \operatorname{sig}=.036)$. Estas últimas correlaciones nos indican, que cuantas menos dificultades interpersonales tenga el adolescente, mejor será su autoconcepto físico y emocional.

\section{EFECTO del Programa de entrenamiento en asertividad y habilidades sociales DEAA}

Antes de exponer el efecto del programa en la muestra elegida, hemos realizado un análisis descriptivo de las variables socio-demográficas y psicológicas del grupo tratamiento y el grupo control. Así, como la realización de pruebas $t$ para muestras independientes.

\section{Variables Socio-Demográficas:}

En primer lugar, se procedió a realizar un análisis descriptivo de las variables sociodemográficas para los grupos de tratamiento y control. Las variables socio-demográficas 
analizadas son: sexo, edad, repetidor, estudios del padre, estudios de la madre, estado civil de los padres y curso (ver tabla 2).

Tabla 2. Variables socio-demográficas: sexo, edad, repetidor, estudios del padre, estudios de la madre, estado civil de los padres y curso, del grupo tratamiento y grupo control.

\begin{tabular}{|c|c|c|c|c|c|}
\hline \multicolumn{2}{|c|}{ Variables socio-demográficas } & \multicolumn{2}{|c|}{ Grupo } & \multirow{3}{*}{$\begin{array}{c}\begin{array}{c}\text { Chi- } \\
\text { cuadrado }\end{array} \\
\\
1.077\end{array}$} & \multirow{3}{*}{$\begin{array}{c}p \\
\\
\\
0.299\end{array}$} \\
\hline & & $\begin{array}{c}\text { Tratamiento } \\
\quad \mathrm{N}=7\end{array}$ & $\begin{array}{c}\text { Control } \\
\mathrm{N}=7\end{array}$ & & \\
\hline SEXO & $\begin{array}{l}\text { Niños } \\
\text { Niñas }\end{array}$ & $\begin{array}{c}0(0 \%) \\
7(100 \%)\end{array}$ & $\begin{array}{l}1(14.29 \%) \\
6(85.71 \%)\end{array}$ & & \\
\hline EDAD & Media (D.T.) & $13(0.58)$ & $13.71(0.95)$ & & \\
\hline CURSO & $\begin{array}{l}1^{\circ} \\
2^{\circ}\end{array}$ & $\begin{array}{l}1(14.28 \%) \\
6(85.75 \%)\end{array}$ & $\begin{array}{l}1(14.28 \%) \\
6(85.75 \%)\end{array}$ & 0.000 & 1.000 \\
\hline REPETIDOR & $\begin{array}{l}\mathrm{Si} \\
\mathrm{No}\end{array}$ & $\begin{array}{c}0(0 \%) \\
7(100 \%)\end{array}$ & $\begin{array}{l}3(42.86 \%) \\
4(57.14 \%)\end{array}$ & 3.81 & $0.051 *$ \\
\hline $\begin{array}{l}\text { ESTUDIOS DEL } \\
\text { PADRE }\end{array}$ & $\begin{array}{c}\text { Primarios } \\
\text { Secundarios } \\
\text { Universitarios }\end{array}$ & $\begin{array}{l}2(28.57 \%) \\
4(57.14 \%) \\
1(14.29 \%)\end{array}$ & $\begin{array}{c}5(71.43 \%) \\
2(28.57 \%) \\
0(0 \%)\end{array}$ & 2.95 & 0.229 \\
\hline $\begin{array}{c}\text { ESTUDIOS DE LA } \\
\text { MADRE }\end{array}$ & $\begin{array}{c}\text { Primarios } \\
\text { Secundarios }\end{array}$ & $\begin{array}{l}2(28.57 \%) \\
5(71.43 \%)\end{array}$ & $\begin{array}{l}3(42.86 \%) \\
4(57.14 \%)\end{array}$ & 0.311 & 0.577 \\
\hline ESTADOS CIVIL & $\begin{array}{c}\text { Casados } \\
\text { Separados/divorcia. }\end{array}$ & $\begin{array}{c}7(100 \%) \\
0(0 \%)\end{array}$ & $\begin{array}{l}6(85.71 \%) \\
1(14.29 \%)\end{array}$ & 1.077 & 0.299 \\
\hline
\end{tabular}


A continuación, se comprobó si ambos grupos estaban igualados, o había alguna diferencia significativa con respecto a las variables socio-demográficas. Para ello, se realizó 1 prueba $t$ para muestras independientes, siendo la VI el grupo (tratamiento Vs control) y la VD, la edad, y 6 pruebas no paramétricas (chi-cuadrado) para las variables sexo, curso, repetidor, estudios del padre, estudios de la madre y estado civil de los padres. Los resultados mostraron que no existían diferencias estadísticamente significativas para las variables edad $[t=-1.698$, $p=.115]$, sexo, curso, estudios del padre, estudios de la madre, y estado civil de los padres. Pero si se observan diferencias significativas para la variable repetidor (ver tabla 2).

\section{Variables Psicológicas:}

\section{○ Análisis Descriptivos}

En lo que respecta a la distribución de la muestra en las variables psicológicas evaluadas, los resultados del análisis descriptivo se recogen en la tabla 3.

Para comprobar si había diferencias entre estos dos grupos, en las variables psicológicas evaluadas, previas al entrenamiento, se realizaron 7 pruebas $t$ para muestras independientes, siendo la VI el grupo (tratamiento Vs control) y las VD, el EHS, el CEDIA, y los cinco factores del AF5. Los resultados muestran (como podemos observar en la tabla 3) que no existen diferencias significativas en la variables conducta asertiva y habilidades sociales (EHS), en la variable de dificultad en las relaciones interpersonales (CEDIA), ni en las dimensiones que miden el autoconcepto: académico/laboral, social, emocional, familiar, y físico. Al comparar las puntuaciones en cada una de las dimensiones del EHS, se encontró significativamente menor la puntuación en la escala de defensa de los propios derechos como consumidor, en la condición experimental [ $t=-2.400, p=.034]$, siendo el grupo control el que obtiene puntuaciones más altas en esta dimensión. En el resto de las escalas del EHS, no se observan diferencias significativas. 
Tabla 3. Media, desviación típica, prueba $t$, significación de las escalas EHS, CEDIA y AF5.

\begin{tabular}{|c|c|c|c|c|c|c|c|}
\hline Escala & Grupo & $\begin{array}{l}\text { Media }(D T) \\
\text { Pretratam. }\end{array}$ & $t$ & $p$ & $\begin{array}{c}\text { Media }(\mathrm{DT}) \\
\text { Postratam }\end{array}$ & $t$ & $p$ \\
\hline \multirow{2}{*}{ EHS } & G. Trata & $77.00(12.22)$ & \multirow[b]{2}{*}{-1.62} & \multirow[b]{2}{*}{.130} & 99.29 (15.99) & \multirow[b]{2}{*}{3.021} & \multirow[b]{2}{*}{$.011 *$} \\
\hline & G. Contr & $85.00(4.55)$ & & & 78.57 (8.56) & & \\
\hline \multirow{2}{*}{ CEDIA } & G. Trata & $60.00(22.11)$ & \multirow[b]{2}{*}{.222} & \multirow[b]{2}{*}{.828} & $46.00(26.97)$ & \multirow[b]{2}{*}{-1.659} & \multirow[b]{2}{*}{.123} \\
\hline & G. Contr & $57.14(25.79)$ & & & $68.00(22.45)$ & & \\
\hline \multicolumn{8}{|l|}{ AF5 } \\
\hline \multirow{2}{*}{ Acad/Lab } & G. Trata & $6.39(2.39)$ & \multirow[b]{2}{*}{-.561} & \multirow[b]{2}{*}{.585} & $6.59(3.21)$ & \multirow[b]{2}{*}{.017} & \multirow[b]{2}{*}{.987} \\
\hline & G. Contr & $7.01(1.65)$ & & & $6.56(1.84)$ & & \\
\hline \multirow{2}{*}{ Social } & G. Trata & $7.56(9.28)$ & \multirow[b]{2}{*}{-.475} & \multirow[b]{2}{*}{.643} & $7.24(1.64)$ & \multirow[b]{2}{*}{-.296} & \multirow[b]{2}{*}{.773} \\
\hline & G. Contr & $7.92(1.73)$ & & & $7.47(1.25)$ & & \\
\hline \multirow{2}{*}{ Emocional } & G. Trata & $3.76(2.55)$ & \multirow[b]{2}{*}{-1.93} & \multirow[b]{2}{*}{.078} & $4.65(1.88)$ & \multirow[b]{2}{*}{-.680} & \multirow[b]{2}{*}{.509} \\
\hline & G. Contr & $6.05(1.83)$ & & & $5.37(2.07)$ & & \\
\hline \multirow{2}{*}{ Familiar } & G. Trata & $8.40(1.20)$ & \multirow[b]{2}{*}{-.158} & \multirow[b]{2}{*}{.877} & $8.35(1.47)$ & \multirow[b]{2}{*}{-.641} & \multirow[b]{2}{*}{.533} \\
\hline & G. Contr & $8.51(1.43)$ & & & $8.82(1.29)$ & & \\
\hline \multirow{2}{*}{ Fisico } & G. Trata & $5.29(1.98)$ & \multirow[b]{2}{*}{-.968} & \multirow[b]{2}{*}{.352} & $5.59(2.52)$ & \multirow[b]{2}{*}{-.677} & \multirow[b]{2}{*}{.511} \\
\hline & G. Contr & $6.20(1.48)$ & & & $6.29(1.13)$ & & \\
\hline
\end{tabular}

Una vez terminado el entrenamiento, y habiéndose obtenido los datos post, se realizó una prueba $t$ para muestras independientes, siendo la VI el grupo (tratamiento Vs control), y las VD las mismas que en las pruebas $t$ anteriores (EHS, CEDIA y AF5). En cuanto la variable de conducta asertiva y habilidades sociales (EHS), se encontró diferencias significativas entre ambos grupos en la puntuación total $[t=3.021, p=.011]$, y en 3 de sus 6 escalas; en decir no y cortar interacciones $[t=3.317, p=.006]$, en hacer peticiones $[t=2.191, p=.049]$, y en iniciar 
interacciones positivas con el sexo opuesto $[t=3.215, p=.007]$. Todas estas diferencias significativas van en la línea de una mayor puntuación para el grupo tratamiento, lo que indica que este presenta, en la evaluación post, una mayor conducta asertiva y habilidades sociales.

En lo que respecta a la variable de dificultad en las relaciones interpersonales (CEDIA), no hay diferencias significativas en la puntuación total, encontrándose la única diferencia significativa en el factor $\mathrm{V}$ relaciones familiares $[t=-2.478, p=.029]$, siendo el grupo control el que presenta puntuaciones más altas, y por lo tanto, mayores dificultades en este tipo de relaciones. Por último, en cuanto a la variable de autoconcepto forma 5 (AF5), no se encuentran diferencias significativas entre ambos grupos en ninguno de los factores: académica/laboral, social, emocional, familiar y física. (Ver tabla 3.)

\section{- Resultados de la aplicación del programa DEAA}

En primer lugar, hemos procedido a comprobar si existen diferencias atribuibles al efecto del tratamiento en las variables psicológicas evaluadas (la conducta asertiva y las habilidades sociales, dificultad en las relaciones interpersonales y el autoconcepto). Para ello, hemos llevado a cabo 7 pruebas $t$ para muestras relacionadas, con dos niveles (pre y post) en ambos grupos (grupo tratamiento y grupo control).

Los datos obtenidos reflejan que solo hay diferencias significativas en la variable de asertividad y habilidades sociales (EHS), tanto para el grupo de tratamiento como para el grupo control, pero no en las otras dos variables, dificultad en relaciones interpersonales (CEDIA) y autoconcepto (AF5). (ver Tabla 4). Además, el grupo de tratamiento obtuvo un alto tamaño del efecto en la variable de habilidades sociales y asertividad. Curiosamente, también observamos un tamaño del efecto moderado en el grupo control en esta misma variable.

Las diferencias significativas reflejadas en la escala EHS para el grupo tratamiento, muestran que ha habido una mejora significativa en asertividad y habilidades sociales tras el 
entrenamiento, sin embargo, las puntuaciones del grupo control para esta misma variable, muestran que ha habido un empeoramiento significativo con el paso del tiempo.

Con respecto al autoconcepto (AF5), aunque no hayan diferencias significativas, el cambio que se refleja en las puntuaciones van en la dirección que se espera, para 3 de las 5 subescalas (académico/laboral, emocional y físico).

Tabla 4. Media, desviación típica, prueba $t$, significación y tamaño del efecto de las escalas de Habilidades Sociales, de Evaluación de Dificultades Interpersonales en la Adolescencia y de Autoconcepto Forma 5.

\begin{tabular}{|c|c|c|c|c|c|c|}
\hline Escalas & Grupo & $\begin{array}{c}\text { Pretratamient } \\
\text { Media }(D T)\end{array}$ & $\begin{array}{c}\text { Postratamient } \\
\text { Media }(D T)\end{array}$ & $\mathbf{t}$ & Sig & $\begin{array}{r}\text { Tamaño } \\
\text { del efecto }\end{array}$ \\
\hline EHS & $\begin{array}{l}\text { G Tratami } \\
\text { G Control }\end{array}$ & $\begin{array}{r}77.00(12.22) \\
85.00(4.55)\end{array}$ & $\begin{array}{r}99.29(15.99) \\
78.57(8.56)\end{array}$ & $\begin{array}{l}-6.25 \\
2.74\end{array}$ & $\begin{array}{l}.001 * \\
.033^{*}\end{array}$ & $\begin{array}{r}-1.58 \\
.98\end{array}$ \\
\hline CEDIA & $\begin{array}{l}\text { G Tratami } \\
\text { G Control }\end{array}$ & $\begin{array}{l}60.00(22.11) \\
57.14(25.79)\end{array}$ & $\begin{array}{l}46.00(26.97) \\
68.00(22.45)\end{array}$ & $\begin{array}{r}1.30 \\
-1.75\end{array}$ & $\begin{array}{l}.240 \\
.131\end{array}$ & $\begin{array}{l}-.44 \\
-.45\end{array}$ \\
\hline AF5 & & & & & & \\
\hline Académica & $\begin{array}{l}\text { G Tratami } \\
\text { G Control }\end{array}$ & $\begin{array}{l}6.39(2.39) \\
7.00(1.65)\end{array}$ & $\begin{array}{l}6.59(3.21) \\
6.56(1.84)\end{array}$ & $\begin{array}{l}-.438 \\
.872\end{array}$ & $\begin{array}{l}.677 \\
.417\end{array}$ & $\begin{array}{l}-.07 \\
.25\end{array}$ \\
\hline Social & $\begin{array}{l}\text { G Tratami } \\
\text { G Control }\end{array}$ & $\begin{array}{l}7.57(0.93) \\
7.92(1.73)\end{array}$ & $\begin{array}{l}7.24(1.64) \\
7.47(1.25)\end{array}$ & $\begin{array}{r}.613 \\
.95\end{array}$ & $\begin{array}{l}.563 \\
.379\end{array}$ & $\begin{array}{l}.26 \\
.30\end{array}$ \\
\hline Emocional & $\begin{array}{l}\text { G Tratami } \\
\text { G Control }\end{array}$ & $\begin{array}{l}3.76(2.55) \\
6.05(1.83)\end{array}$ & $\begin{array}{l}4.65(1.88) \\
5.37(2.07)\end{array}$ & $\begin{array}{c}-1.044 \\
.927\end{array}$ & $\begin{array}{l}.337 \\
.390\end{array}$ & $\begin{array}{l}.05 \\
.35\end{array}$ \\
\hline Familiar & $\begin{array}{l}\text { G Tratami } \\
\text { G Control }\end{array}$ & $\begin{array}{l}8.40(1.20) \\
8.52(1.43)\end{array}$ & $\begin{array}{l}8.35(1.47) \\
8.82(1.29)\end{array}$ & $\begin{array}{c}.157 \\
-.555\end{array}$ & $\begin{array}{r}.88 \\
.599\end{array}$ & $\begin{array}{l}.04 \\
.22\end{array}$ \\
\hline Física & $\begin{array}{l}\text { G Tratami } \\
\text { G Control }\end{array}$ & $\begin{array}{l}5.30(1.98) \\
6.20(1.48)\end{array}$ & $\begin{array}{l}5.59(2.52) \\
6.29(1.13)\end{array}$ & $\begin{array}{l}-.58 \\
-.258\end{array}$ & $\begin{array}{l}.583 \\
.805\end{array}$ & $\begin{array}{l}-.13 \\
.07\end{array}$ \\
\hline
\end{tabular}

Para el CEDIA, aunque no haya diferencias significativas en la puntuación total, y en ninguna de las subescalas que lo forman, si que se aprecia un cambio en las puntuaciones, que 
van en la dirección de la mejora. En 3 de los 5 factores que forman el CEDIA (factor I aserción, factor II relaciones con el otro sexo, y factor IV hablar en público), la puntuación Media pre del grupo tratamiento era superior a la del grupo control, lo que indica una mayor dificultad en las relaciones interpersonales. Sin embargo, en la puntuación Media post del grupo tratamiento, en estos mismos factores, es inferior a la del grupo control, lo que nos indica que el grupo tratamiento tiene menos dificultades en las relaciones interpersonales después del entrenamiento que el control. Las puntuaciones de los otros dos factores del grupo tratamiento (factor III relaciones con iguales y factor IV relaciones familiares), eran inferiores en la evaluación pre, y se mantienen inferiores en la evaluación post (ver tabla 5).

Tabla 5. Medias, desviaciones típicas, diferencias pre-post, nivel de significación y tamaño del efecto en el grupo tratamiento en los factores del Cuestionarios de Evaluación de Dificultades Interpersonales en la adolescencia.

\begin{tabular}{|l|c|r|r|r|r|c|}
\hline \multicolumn{1}{|c|}{$\begin{array}{c}\text { Subescalas } \\
\text { CEDIA }\end{array}$} & Grupo & $\begin{array}{c}\text { Pretratamient } \\
\text { Media }(\text { DT })\end{array}$ & $\begin{array}{c}\text { Postratamien } \\
\text { Media }(\boldsymbol{D T})\end{array}$ & $\boldsymbol{t}$ & Sig & $\begin{array}{c}\text { Tamaño } \\
\text { del efecto }\end{array}$ \\
\hline $\begin{array}{l}\text { Factor I } \\
\text { aserción }\end{array}$ & G.Tratam & $29.29(12.49)$ & $21.57(9.59)$ & 1.325 & .233 & 0.71 \\
\hline $\begin{array}{l}\text { Factor II } \\
\text { relaciones con } \\
\text { el otro sexo. }\end{array}$ & G. Contro & $24.86(9.20)$ & $30.28(8.77)$ & -2.465 & $\mathbf{. 0 4 9 *}$ & $\mathbf{- 0 . 6}$ \\
\hline $\begin{array}{l}\text { Factor III } \\
\text { relaciones } \\
\text { iguales }\end{array}$ & G. Contro & $12.71(6.68)$ & $15.14(4.41)$ & -1.626 & .155 & -0.44 \\
\hline $\begin{array}{l}\text { Factor IV } \\
\text { hablar en } \\
\text { publico }\end{array}$ & G. Contro & $5.71(4.64)$ & $6.86(5.79)$ & -.584 & .581 & -0.22 \\
\hline $\begin{array}{l}\text { Factor V } \\
\text { relaciones } \\
\text { familiares }\end{array}$ & G. Tratam & $10.57(4.72)$ & $6.71(5.15)$ & 1.983 & .095 & 0.78 \\
\hline
\end{tabular}


Además, si comparamos todas las Medias pre y post del grupo tratamiento, apreciamos como ha habido un descenso de las puntuaciones en todos los factores del CEDIA, excepto en el factor III relaciones con los iguales. Sin embargo, para el grupo control, las Medias pre son más bajas que las post. Por lo tanto, esto nos indica que el grupo que ha recibido tratamiento ha mejorado en las relaciones interpersonales, y el grupo control, por el contrario, ha empeorado. Sin llegar a ser ambos resultados significativos. (Ver tabla 5).

Tabla 6. Medias, desviaciones típicas, diferencias pre-post, nivel de significación y tamaño del efecto en el grupo tratamiento en los factores de la Escala de Habilidades Sociales.

\begin{tabular}{|c|c|c|c|c|c|c|}
\hline $\begin{array}{c}\text { Subescalas } \\
\text { EHS }\end{array}$ & Grupo & $\begin{array}{l}\text { Pretratamien } \\
\text { toMedia }(D T)\end{array}$ & $\begin{array}{c}\text { Postratamient } \\
\text { o Media }(D T)\end{array}$ & $t$ & Sig & $\begin{array}{r}\text { Tamaño } \\
\text { del efecto }\end{array}$ \\
\hline $\begin{array}{l}\text { Autoexpresió } \\
\text { n }\end{array}$ & $\begin{array}{l}\text { G.Tratam } \\
\text { G. Contro }\end{array}$ & $\begin{array}{c}19.43(4.68) \\
21.29(1.25)\end{array}$ & $\begin{array}{l}25.29(5.28) \\
21.57(3.36)\end{array}$ & $\begin{array}{l}-4.866 \\
-.24\end{array}$ & $\begin{array}{c}. \mathbf{0 0 3} * \\
.818\end{array}$ & $\begin{array}{r}-1.18 \\
-.12\end{array}$ \\
\hline $\begin{array}{l}\text { Defensa } \\
\text { Derechos }\end{array}$ & $\begin{array}{l}\text { G. Tratam } \\
\text { G. Contro }\end{array}$ & $\begin{array}{r}10.86(3.48) \\
14.57(2.15)\end{array}$ & $\begin{array}{r}14.43(2.07) \\
12.29(2.87)\end{array}$ & $\begin{array}{l}-3.99 \\
1.804\end{array}$ & $\begin{array}{l}. \mathbf{0 0 7} * \\
.121\end{array}$ & $\begin{array}{c}\mathbf{- 1 . 2 9} \\
.91\end{array}$ \\
\hline Decir No & $\begin{array}{l}\text { G. Tratam } \\
\text { G. Contro }\end{array}$ & $\begin{array}{r}11.57(4.58) \\
13.86(3.24)\end{array}$ & $\begin{array}{r}18.57(4.24) \\
12.57(2.23)\end{array}$ & $\begin{array}{l}-5.07 \\
1.02\end{array}$ & $\begin{array}{l}.002 * \\
.349\end{array}$ & $\begin{array}{r}\mathbf{- 1 . 5 9} \\
.47\end{array}$ \\
\hline $\begin{array}{l}\text { Hacer } \\
\text { Peticiones }\end{array}$ & $\begin{array}{l}\text { G. Tratam } \\
\text { G. Contro }\end{array}$ & $\begin{array}{l}14.29(1.99) \\
14.00(2.77)\end{array}$ & $\begin{array}{r}14.42(2.07) \\
11.57(2.76)\end{array}$ & $\begin{array}{l}-.240 \\
1.68\end{array}$ & $\begin{array}{l}.818 \\
.144\end{array}$ & $\begin{array}{l}-.06 \\
.88\end{array}$ \\
\hline $\begin{array}{l}\text { Iniciar } \\
\text { Interacciones }\end{array}$ & $\begin{array}{l}\text { G. Tratam } \\
\text { G. Contro }\end{array}$ & $\begin{array}{l}11.29(2.56) \\
11.57(2.23)\end{array}$ & $\begin{array}{r}14.43(2.70) \\
10.00(2.45)\end{array}$ & $\begin{array}{l}-2.66 \\
1.58\end{array}$ & $\begin{array}{l}. \mathbf{0 3 8 *} \\
.166\end{array}$ & $\begin{array}{c}\mathbf{- 1 . 1 9} \\
.67\end{array}$ \\
\hline $\begin{array}{l}\text { Expresar } \\
\text { Enfado }\end{array}$ & $\begin{array}{l}\text { G. Tratam } \\
\text { G. Contro }\end{array}$ & $\begin{array}{r}9.57(3.31) \\
9.71(3.04)\end{array}$ & $\begin{array}{r}12.14(3.08) \\
10.57(1.72)\end{array}$ & $\begin{array}{l}-1.76 \\
-.969\end{array}$ & $\begin{array}{l}.129 \\
.37\end{array}$ & $\begin{array}{l}-.80 \\
-.36\end{array}$ \\
\hline
\end{tabular}

En segundo lugar, se analizaron las puntuaciones pre-post en los diferentes factores de la escala de Habilidades Sociales obtenidas por ambos grupos, utilizando una prueba de 
diferencia de medias para medidas repetidas $t$ de Student. Los datos indican que solo hubo diferencias significativas para el grupo tratamiento, en las siguientes escalas: autoexpresión en situaciones sociales $(t=-4.87, p=.003)$, defensa de los propios derechos como consumidor $(t=-$ 3.99, $p=.007)$, decir no y cortar interacciones $(t=-5.07, p=.002)$, e iniciar interacciones positivas con el sexo opuesto $(t=-2.66, p=.038)$, no encontrándose diferencias significativas en el factor de expresión de enfado o disconformidad $(t=-1.76, p=.129)$, ni en el de hacer peticiones $(t=-.240, p=.818)$. En el grupo control no se encontró ninguna diferencia significativa. (ver Tabla 6). Podemos observar que el mayor tamaño del efecto se encuentra en las subescalas de decir no, defensa de los derechos y autoexpresión. En el grupo control, encontramos un tamaño del efecto moderado en las subescalas de defensa de los derechos y hacer peticiones.

\section{DISCUSIÓN}

Los objetivos principales de este estudio han sido, por un lado, evaluar diferentes variables (socios-demográficas y psicológicas) en una serie de alumnos, pertenecientes a dos IES distintos, y por otro, la creación de un programa de habilidades sociales/asertividad, a partir de las necesidades detectadas en el alumnado, y la valoración de los efectos del mismo. Las variables utilizadas para medir los beneficios del programa, son la conducta asertiva y habilidades sociales, el nivel de dificultad en las relaciones interpersonales, y el autoconcepto. A la vista de los resultados obtenidos, se ven confirmadas parte de las hipótesis que planteábamos al inicio de esta investigación. Estos resultados están en consonancia con los obtenidos en otros estudios, que encuentran efectos positivos de programas similares en la conducta social de los adolescentes (Llanos Baldivieso, 2006; Rosa et al., 2002).

En primer lugar, tal y como planteábamos en la hipótesis I, encontramos una alta relación negativa entre la variable asertividad/hhss, y la variable de dificultades 
interpersonales. Por lo tanto, los alumnos que hacen gala de un comportamiento asertivo y habilidades sociales, son los que, durante la adolescencia, tienen menos dificultades en las relaciones interpersonales.

En lo que se refiere a la segunda hipótesis de partida, la adolescencia es una época de la vida donde las relaciones sociales se tornan de los aspectos más importantes, incluso en algunos casos, adquiere más relevancia que la familia. Por lo que, el sentirse queridos y aceptados dentro de un grupo de iguales (para lo que tener una buenas habilidades sociales se considera importante) podría influir en el autoconcepto del adolescente sobre sí mismo como una persona hábil, con capacidad de desenvolverse de forma positiva en diversos situaciones sociales de su vida. Esto es lo que proponía nuestra segunda hipótesis, y es lo que los datos muestran, en parte. Si que habría un relación significativa entre tener menos dificultades interpersonales y mejor autoconcepto emocional y físico, pero, aunque los resultados vayan en el camino esperado, no hay correlación significativa entre dificultades interpersonales y el autoconcepto social, académico/laboral, ni familiar.

Siguiendo con esta segunda hipótesis, solo se encuentra relación significativa entre, tener más asertividad/hhss y un autoconcepto emocional más alto. No mostrando los datos ninguna relación significativa entre esta variable, y el resto de las dimensiones del autoconcepto, por lo que habría poca relación entre comportarnos de manera asertiva y poseer buenas habilidades sociales, y tener un autoconcepto alto.

Un dato importante encontrado en nuestro estudio, es el porcentaje de adolescentes que presentan puntuaciones bajas en hhss (el $42.31 \%$ están por debajo de percentil 30 y el $64 \%$ por debajo del percentil 50 en la Escala EHS). A su vez, estos datos se ven corroborados por las puntuaciones medias en dificultades en las relaciones interpersonales (evaluado a través del CEDIA): la media del estudio original $($ Media $=38.37$; D.T.= 18.05) (Inglés Saura, Méndez e Hidalgo, 2000), es bastante más baja que la media de nuestro estudio (Media $=50.73$; D.T.= 
22.16). Esto datos avalan la necesidad de implementar programas dirigidos a prevenir posibles problemas futuros en nuestros adolescentes.

Con respecto a la hipótesis III, tal y como se esperaba, encontramos cambios estadísticamente significativos en la escala EHS, seleccionada para medir el efecto del entrenamiento. Según los datos, los adolescentes que habían seguido el programa, habían mejorado significativamente en asertividad/habilidades sociales. Encontrándose, además, un gran tamaño del efecto entre ambas medidas pre-post en la escala EHS. En lo que respecta a las subescalas de este autoinforme, observamos una mejora significativa en 4 de las 6 que constituyen el instrumento: autoexpresión en situaciones, defensa de los propios derechos como consumidor, decir no y cortar interacciones e iniciar interacciones positivas con el sexo opuesto.

En referencia al grupo control, no solo no se ha encontrado que este grupo no haya mejorado en habilidades sociales, sino que se ha visto un empeoramiento importante con el paso del tiempo, tal y como muestran las pruebas $t$ para muestras relacionadas, donde hay una diferencia significativa. Estos datos dan más fuerza a conclusiones de autores como DíazBarriga y Hernández (2001), que manifiestan la necesidad de desarrollar programas trasversales dentro de los contenidos del currículum escolar, además de fomentar y trabajar estrategias adecuadas para el comportamiento social de los alumnos en sus respectivas aulas. Si no se hace, corremos el riesgo de empeorar la conducta de los alumnos a lo largo del ciclo escolar, tal y como se muestra en esta y otras investigaciones (Llanos Baldivieso, 2006).

En cuanto a las demás variables utilizadas para medir la mejora del entrenamiento en habilidades sociales, los datos no muestran ningún cambio significativo. En la variable de dificultades en las relaciones interpersonales (CEDIA), aunque no haya mejora significativa, si que los datos van en este camino. Como se observa, la puntuación del grupo tratamiento en la evaluación post es menor que en la pre, lo que indica que ha habido una disminución en las 
dificultades que encuentran los alumnos al relacionarse a nivel social. Por el contrario, para el grupo control, las puntuaciones post son mayores, lo que indica un aumento de las dificultades en las relaciones interpersonales, aunque sin llegar a ser significativo este empeoramiento.

Al realizar la comparación inter-grupal, se observa como las puntuaciones pre del grupo tratamiento son superiores a las del grupo control, lo que muestra una mayor dificultad en las relaciones sociales. Cuando posteriormente, se aplicó el entrenamiento y se volvió a evaluar, se ve como estas puntuaciones del grupo tratamiento, disminuyeron por debajo de las del grupo control, lo que indica, que tras el entrenamiento, el grupo tratamiento tiene menos dificultades en las relaciones interpersonales que el control. Este cambio de las puntuaciones se reproduce en 3 de las 5 subescalas de las que está formado el CEDIA (Factor I aserción, Factor II relaciones con el otro sexo, y Factor IV hablar en público). Para las otras dos subescalas, el grupo tratamiento tenía menos dificultades en las puntuaciones pre, y sigue teniendo menos en las post (Factor III relaciones con los iguales y Factor V relaciones familiares). Por lo tanto, esta hipótesis III, se cumple en parte.

En los que respecta a la última hipótesis, la cuarta, los datos no van en la dirección esperada. Los resultados de la evaluación post del autoconcepto, no muestran una mejora significativa del grupo tratamiento tras el entrenamiento. Dado que hemos aplicado un programa para mejorar las habilidades sociales, llama la atención que de las 5 dimensiones del autoconcepto, la dimensión social no haya salido significativa, y que los datos vayan justo al contrario (un menor autoconcepto). Pero si analizamos los ítems que componen esta dimensión, de los 6 ítems, 4 están relacionados con la facilidad para hacer amigos/ser amigable. Probablemente, la formación del autoconcepto y su cambio sean procesos más lentos, que necesitan mucho más tiempo y por lo tanto estos cambios -en el caso de que se produjeran- dado el momento en el que fueron evaluados los alumnos, no los podríamos detectar. 
Haciendo referencia a los datos obtenidos de forma pre y post al entrenamiento, podemos concluir que el entrenamiento en asertividad/habilidades sociales, ha podido contribuir dotando a los alumnos de herramientas sobre cómo actuar de forma adecuada, en las diferentes situaciones sociales en las que presentan déficits. Estos conocimientos se transforman en una mejora significativa en las puntuaciones de la escala EHS.

El haber adquirido este conocimiento, les ha llevado a disminuir ciertas dificultades en las relaciones sociales, aquellas con las que se han encontrado en el poco tiempo que ha durado el entrenamiento. Sin embargo, probablemente ha de pasar algún tiempo, hasta que se les presenten otras situaciones sociales, y así, poder poner en práctica sus herramientas adquiridas. De ahí que el cuestionario que indica las dificultades interpersonales muestre una disminución de estas dificultades, sin llegar a ser significativas, para lo cual, sería necesario el paso del tiempo.

Por último, la variable del autoconcepto, es la que se presenta como más resistente al cambio. El concepto que una persona tiene de sí misma se forma a lo largo de los años, y a través de las interacciones y situaciones en las que esta ha estado inmersa. Por lo tanto, su cambio se esperaría cuando estos adolescentes se hayan enfrentado varias veces a dificultades sociales, y hayan sabido cómo actuar de forma correcta. Además, en el autoconcepto están influyendo multitud de factores, para lo que las habilidades sociales supondrían un granito de arena en su mejora.

Como conclusión, los datos obtenidos en esta investigación, coinciden en su globalidad con los resultados de meta-análisis realizados sobre esta materia en cuestión. Esta recopilación de estudios, dan a la luz la eficacia de estos programas de asertividad y habilidades sociales, eficacia que se ve incrementada con el tiempo (Beelmann et al., 1994; Denham y Almeida, 1987; Schneider, 1992; Rosa et al., 2002), que es lo que se esperaría si se volviera a evaluar pasado un tiempo a esta muestra de alumnos. Estos autores, apuntan que las variables en las 
que más se refleja el éxito de estos entrenamientos, son las variables de competencia social (asertividad, afrontamiento y habilidades sociales), frente a variables más periféricas (ansiedad y autoestima), tal y como reflejan los resultados de esta investigación.

A pesar de que los datos encontrados son esperanzadores, existen una serie de limitaciones metodológicas que convendrían resolver en trabajos futuros, para de este modo generalizar los resultados y obtener mayor relevancia científica. Una de estas limitaciones se refiere al carácter inmediato de esta investigación, lo que conlleva que los resultados aquí mostrados pertenecen a un análisis preliminar de la aplicación del programa. Sin duda, sería interesante recoger y analizar datos que muestren la generalización de lo adquirido con el tratamiento a lo largo del tiempo, para comprobar si los resultados de esta evaluación muestran una mejora significativa de las variables que no han mostrado cambios significativos. Además de señalar si los cambios logrados se siguen manteniendo.

Otra de las mayores limitaciones con las que nos hemos encontrado en el estudio, es la muestra, ya que no ha sido lo suficientemente grande como para considerar estos resultados generalizables. A esto se le une, que el objetivo con el que ha sido creado este programa de entrenamiento en asertividad y habilidades sociales DEAA, es para que se utilice en clase de tutoría, y realizado por los tutores. Por lo que futuros estudios que evalúen la efectividad de este programa, deben tener en cuenta el desarrollo del mismo dentro de este contexto.

Con respecto a las escalas seleccionadas, en concreto la AF5, comentan los autores (Goraigordobil, Durá y Pérez, 2005) que la evaluación del aspecto cognitivo del conocimiento de uno mismo (autoconcepto), conlleva aspectos valorativos y afectivos de la persona (autoestima). Por esta razón, la evaluación del autoconcepto conllevaría, en parte, la evaluación de la autoestima de los alumnos. Sin embargo, consideramos que para estudios futuros sería bueno que se utilizara una escala que evaluara directamente la autoestima, y así comprobar el efecto de este entrenamiento sobre esta variable. 
En resumen, este estudio supone un primer acercamiento a la valoración objetiva de creación de un programa de entrenamiento en asertividad y habilidades sociales para adolescentes, con una serie de características particulares. Ya que se trata de un programa creado por los autores de este trabajo, sería muy recomendable ahondar en el estudio de la eficacia de este entrenamiento, para crear una versión más depurada y ajustada al contexto para el que fue diseñado. En definitiva, podemos señalar que, los resultados muestran los beneficios que acciones como estas tienen en adolescentes con problemas de relaciones sociales.

\section{REFERENCIAS}

-Argyle, M. (1969). Social Interaction. Londres: Methuen.

-American Psychiatric Association (2000). Diagnostic and statistical manual of mental disorders (4 $4^{\mathrm{a}}$ ed. texto revisado). Washington, DC.

-Bandura, A. (1982). Teoría del aprendizaje social. Madrid: Espasa-Calpe.

-Beelmann, A., Pfingsten, U. y Losel, F. (1994). Effects of training social competence in children: a meta-analysis of recent evaluation studies. Journal of Clinical Child Psychology, 23, 260-271.

-Bowlby, J. (1979). The making and breaking of affectional bonds. Londres: Tavistock.

-Brennan, T. (1982). Loneliness at adolescence. In Peplau, L.A., and Perlman, D. (Eds.), Loneliness: A Sourcebook of Current Theory, Research and Therapy. New York: WileyInterscience.

-Buck, R., (1991). Temperament, social skills, and the communication of emotion: A developmental-interactionist view. En D.G. Gilbert, y J.J. Connolly, (Eds.), Personality, Social Skills, and Psychopathology. An Individual Differences Approach (pp. 85-106). New York: Plenum Press.

-Caballo, V.E. (1989). La multidimensionalidad conductual de las Habilidades Sociales: propiedades psicométricas de una nueva medida de autoinforme. Comunicación presentada en las VII Jornadas de Modificación de Conducta: Habilidades Sociales, Madrid, 3 al 6 de mayo. -Caballo, V.E. y Buela, G. (1988). Factor analyzing the College Self-Expression Scale with a Spanish population. Psychological Reports, 63, 503-507.

-Caballo, V.E. (2009) Manual de evaluación y entrenamiento de las habilidades sociales, Madrid: Siglo XXI.

-Camacho Gómez, C. y Camacho Calvo, M. (2005). Habilidades sociales en adolescencia: un programa de intervención. Revista Profesional Española de Terapia Cognitivo-Conductual, 3, $1-27$.

-Carrasco, I., Clemente, M. y Llavona, L. (1983). Análisis de componentes básicos del Inventario de asertividad de Rathus. Revista Española de Terapia del Comportamiento, 1, 
249-264.

-Chou, K-L. (2000). Intimacy and psychosocial adjustment in Hong Kong Chinese adolescents. Journal of Genetic Psychology, 161, 141-152.

-Christoff, K.A., Scott, W.O.N., Kelley, M.L., Schlundt, D., Baer, G. y Kelly, J.A. (1985). Social skills and social problem-solving training for shy young adolescents. Behavior Therapy, $16,468-477$.

-Denham, S. y Almeida, M.C. (1987). Children’s social problem-solving skills, behavioral adjustement, and interventions: A meta-analysis evaluating theory and practice. Journal of Applied Developmental Psychology, 8, 391-409.

-Díaz-Barriga, F. y Hernández, G. (2001). Estrategias docentes para un aprendizaje significativo. México DF: McGraw-Hill.

-Erikson, E.H. (1963). Childhood and society. New York: Norton.

-Fernández Berrocal, P. y Melero Zabal, M.A. (1995). La interacción social en contextos educativos. Madrid: Siglo XXI.

-Flores, M.F., y Díaz. R.D. (1995). Desarrollo y validación de una Escala Multidimensional de Asertividad para Estudiantes. Revista Mexicana de Psicología, 12, 133-144. -Francis, G., Last, C.G., y Strauss, C. C. (1992). Avoidant disorder and social phobia in children and adolescents. Journal of the American Academy of Child and Adolescent Psychiatry, 31,1086-1089.

-Francis, G., y Radka, D.F. (1995). Social anxiety in children and adolescents. En M.B. Stein (Ed.), Social phobia: Clinical and research perspectives (pp. 119-143). New York: Guildford Press.

-Frederick y Morgeson. (2005). Selecting individuals in team settings: the importance of social skills, personality characteristics, and teamwork knowledge. Personnel Psychology, 58, 583611.

-Galassi, J.P. y Galassi, M.D. (1980). Similarities and differences between two assertion measures: Factor analysis of the College Self-Expression Scale and the Rathus Assertiveness Schedule. Behavioral Assessment, 2, 43-57.

-Garaigordobil Landazabal, M. (1996). Evaluación de una intervención psicoeducativa en sus efectos sobre la conducta prosocial y la creatividad. Madrid: Edita.

-Garaigordobil Landazabal, M. (2001). Intervención con adolescentes: impacto de un programa en la asertividad y en las estrategias cognitivas de afrontamiento de situaciones sociales. Psicología Conductual, 9, 221-246.

-Garaigordobil Landazabal, M., Durá, A., \& Pérez, J. I. (2005). Síntomas psicopatológicos, problemas de conducta y autoconcepto-autoestima: Un estudio con adolescentes de 14-17 año. Anuario de Psicología Clínica y de la Salud,1 , 53-63.

-García Pérez, E. y Magaz Lago, A. (1998). Ratones, Dragones y Seres Humanos Auténticos. Manual de entrenamiento asertivo: Estrategias para aumentar la autoestima de jóvenes y adolescentes. Cruces-Barakaldo: Grupo Albor COHS.

-García, F. y Musitu, G. (1999). AF5 Autoconcepto Forma 5. Madrid: Tea.

-García, J.F., Musitu, G. y Veiga, F. (2006). Autoconcepto en adultos de España y Portugal. Psicothema, 18, 551-556.

-Gil, F. y León, J.M. (1998). Habilidades Sociales. Teoría, Investigación e intervención. Madrid: Sintesis.

-Gilbert, E. y DeBlassie, R. (1984). Anorexia nervosa: adolescent starvation by choice. 
Adolescence, 19, 839-846.

-Gismero González, E. (2000). EHS Escala de Habilidades Sociales. Madrid: Tea. -Hansen, D. J., St. Lawrence, J. S., \& Christoff, K. A. (1988). Conversational skills of inpatient conduct disordered youths: Social validation of component behaviors and implications for skills training. Behavior Modification, 12, 424-444.

-Hansen, D. J., Giacoletti, A. M., \& Nangle, D. W. (1995). Social interactions and adjustment. In V. B. Van Hasselt \& M. Hersen (Eds.), Handbook of adolescent psychopathology: A guide to diagnosis and treatment (pp.102-129). New York: MacMillan

-Hansen, D.J., Nangle, D.W. y Meyer, K.A. (1998). Enhancing the effectiveness of social skills interventions with adolescents. Education and Treatment of Childre, 21, 489-513. -Harris, J. R. (1995). Where is the child's environment? A group socialization theory of development. Psychological Review, 102, 458-489.

-Inglés Saura, C.J., Méndez, F.X. e Hidalgo, M.D. (2000). Cuestionario de Evaluación de Dificultades Interpersonales en la Adolescencia. Psycothema, 12, 390-398.

-Inhelder, B. y Piaget, J. (1996). De la lógica del niño a la lógica del adolescente. Barcelona: Paidós.

-Kaiser, C. y Berndt, D. (1985). Predictors of loneliness in the gifted adolescent. Gifted Child Quart, 29, 74-77.

-Kohlberg, L. (1973). Continuities in childhood and adult moral development revisited. En P.B. Baltes y K.W. Schaie (Eds.), Life span developmental psychology: Personality and socialisations. New York: Academic Press.

-Lange, A.J., Rimm, D.C. y Loxley, J., (1978). Cognitive-behavioral assertion training procedures. Approaches to assertion training. Monterry: Brooks/Cole. -Llanos Baldivieso, C.C. (2006). Efectos de un programa de enseñanza en habilidades sociales. Tesis Doctoral. Universidad de Granada. -Monjas, I. (2002). Programa de enseñanza de habilidades de interacción social (PEHIS). Madrid: Ciencias de la Eduación Preescolar y Especial.

-Monjas, I. (2009). Cómo promover la convivencia: Programa de asertividad y habilidades sociales (PAHS). Educación Infantil, Primaria y Secundaria. Madrid: CEPE

-Moore, D. y Schultz, N.R. (1983). Loneliness and adolescence: Correlates, attributions, and coping. Journal of Youth and Adolescence, 12, 95-100.

-Morgenson, F.P., Reidor, M.H. y Campion, M.A. (2005). Selecting individuals in team settings: the importance of social skills, personality characteristics, and teamwork knowledge. Personnel psychology, 58, 583-611.

-Musitu, G. y Cava, M.J. (2003). El rol del apoyo social en el ajuste de los adolescentes. Intervención Psicosocial, 12, 179-192.

-Nas, C., Brugman, D. y Koops, W. (2005). Effects of the EQUIP programme on the moral judgement, cognitive distortions, and social skills of juvenile delinquents. Psychology, Crime and Law, 11, 421-434.

-Openshaw, D.K., Mills, T.A., Adams, G.R., y Durso, D.D. (1992). Conflict resolution in parent-adolescent dyads: The influence of social skills training. Journal ofAdolescent Research, 7, 457-468.

-O'Connor, R. D. (1969). Modification of social withdrawal through symbolic modeling. Journal of Applied Behavior Analysis, 2, 15-22.

-Rosa, A.I., Inglés, C.J., Olivares, J., Espada, J.P., Sánchez-Meca, J. y Méndez, X. (2002). 
Eficacia del entrenamiento en habilidades sociales con adolescentes: de menos a más. Psicología Conductual, 10, 543-561.

-Riesco González, M. (2005). Habilidades sociales en adolescentes con problemas de desadaptación social. Estudio diagnóstico y propuesta de intervención. Disponible en http://www.cesdonbosco.com/revista/articulos2005/diciembre05/manuelriesco.pdf

-Roca, E. (2007). Cómo mejorar tus habilidades sociales. Programa de asertividad, autoestima e inteligencia emocional. Valencia: ACDE

-Sánchez-Queija, I. y Oliva, A.(2003). Vínculos de apego con los padres y relaciones con los iguales durante la adolescencia. Revista de Psicología Social, 18, 71-86. -Schneider, B.H. (1992). Didactic methods for enhancing children's peer relations: a quantitative review. Clinical Psychology Review, 12, 363-382.

-Shavelson, J., Hubner, J.J. y Stanton, G.C. (1976). Self-concept: validation of construct interpretations. Review of Educational Research, 46, 407-442.

-Tabachnick, N. (1981). The interlocking psychologies of suicide and adolescence. Adolescent Psychiatry, 9, 399-410.

-Tomás, J.M. y Oliver, A. (2004). Análisis Psicométrico Confirmatiorio de una Medida Multidimensional del Autoconcepto en Español. Revista Internacional de Psicología, 38, $285-$ 293.

-Vaello Orts, J. (2005). Las habilidades sociales en el aula. Madrid: Santillana. -Vallés Arándiga, A. y Vallés Tortosa, C. (1994). Cuaderno para mejorar las habilidades sociales, autoestima y solución de problemas (III).Nivel óptimo: educación secundaria, obligatoria, BUP, FP,... Madrid: EOS

-Vera Guerro, M. N. y Roldán Maldonado, G. M. (2009) Ansiedad social. Manual práctico para superar el miedo. Madrid: Ed. Pirámide.

-Walters, K.S., e Inderbitzen, H.M. (1998). Social anxiety and peer relations among adolescents: Testing a psychobiological model. Journal of Anxiety Disorders, 12, 183-198. -Wilkinson, J. y Canter, S. (1982). Social skills training manual: Assessment, programme design and management of training, Chichester: Wiley. 


\section{ANEXO I: Planificación del entrenamiento: \\ PRIMERA SESIÓN}

Presentación del Psicólogo y de cada uno de los miembros del grupo (nombre, edad, procedencia, hobbies, qué esperan del programa, etc.). Objetivos del programa y duración.

Temas a tratar: -Explicación de las habilidades sociales, importancia, implicación, etc.

-Comunicación verbal y no verbal. Utilización de role-play para ejemplificar la no verbal.

-Explicación de los 3 estilos de conducta social. Ejemplificarlos mediante 3 personales de Los Simpsons.

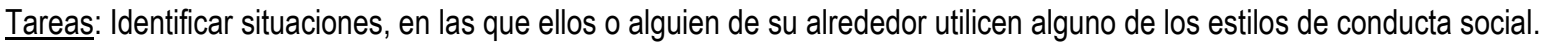

\section{SEGUNDA SESIÓN}

Comentar tarea y repasar contenido de la sesión anterior: role-play de los diferentes estilos de conducta social (todos participan)

Temas a tratar: -Explicar la relación Situación-Pensamiento-Emoción-Conducta. Utilizar un ejemplo donde la misma persona, en la misma situación, se siente y responde de forma distinta. Destacar que no es la situación la que hace que nos sintamos de una forma u otra, sino cómo interpretemos la situación. Ejercicio de la Joven y la Anciana.

Tareas: Durante la semana, cuando se produzca alguna situación donde se sientan mal, que la reinterpreten y la valoren desde un punto de vista distinto.

\section{TERCERA SESIÓN}

Comentar tarea de la sesión anterior.

Temas a tratar: -Componentes de la asertividad: Escucha activa y empatía. Hacer role-playing con los alumnos, de situaciones en las que hay y no escucha activa y empatía.

-Derechos asertivos: Ver la lista de derechos más importantes. Todos van ejemplificados, y para algunos, se utiliza dinámicas y role-playing para representarlos, además de fotografías representativas en las transparencias.

Tareas: Ser consciente, a lo largo de la semana, de situaciones en las que respeto algún derecho propio o de terceros, o en la que no lo hago.

\section{CUARTA SESIÓN}

Comentar tarea de la sesión anterior. Revisar contenido pasado: -tramo de película (Te Doy Mis Ojos) donde se violan los derechos de unas personas, para que los alumnos reflexionen sobre los derechos que no están siendo respetados. -Tramo de un capítulo de Los Simpsons, donde Waylon Smithers no se respeta.

Temas a tratar: -La habilidad de Decir No: Debatir ventajas de decir No e inconvenientes de decir, siempre a todo y a todos, si.

-Formas asertivas de decir no: *Técnica del disco rayado, *Técnica de aplazar la respuesta, *Técnica de aliarse con el enemgio. Dinámica de las tres técnicas.

-Hacer peticiones de forma asertiva: *Técnica de inversión, *Técnica de pie en la puerta.

Tareas: Ser capaces de decir no cuando alguien nos pide algo que no deseamos hacer, pero de forma asertiva. Hacer alguna petición. 
Repasar tareas de la sesión anterior.

Temas a tratar: -Hacer y recibir críticas. Hacer role-playing donde se hace una crítica "agresiva", y así descubran cómo se hace sentir a las persona, y la vean la importancia de hacerlas de forma asertiva.

-Formas de hacer una crítica asertiva: *Técnica del sándwich

-Formas de responder a una crítica adecuadamente.

-Hacer y recibir cumplidos: Ver la importancia de hacer cumplidos mediante ejemplos.

Formas de hacer cumplidos, y de recibirlos.

Tareas: Hacer algún cumplido a alguien de nuestra familia, y ver su reacción. Hacer alguna crítica de forma asertiva.

\section{SEXTA SESIÓN}

Repasar tarea de la sesión anterior.

Temas a tratar: -Resolución de conflictos= Tipos de conflictos (competitivos Vs intereses compartidos).

-Responder asertivamente a los conflictos: *Negociación, *Técnica de resolución de conflictos.

Despedida. 